LEADING ARTICLE

\title{
Food allergy in irritable bowel syndrome: new facts and old fallacies
}

\author{
E Isolauri, S Rautava, M Kalliomäki
}

Gut 2004;53:1391-1393. doi: 10.1136/gut.2004.044990

The notion of food allergy in irritable bowel syndrome (IBS) is not new. However, recent evidence suggests significant reduction in IBS symptom severity in patients on elimination diets, provided that dietary elimination is based on foods against which the individual had raised $\lg G$ antibodies. These findings should encourage studies dissecting the mechanisms responsible for lgG production against dietary antigens and their putative role in IBS

See end of article for authors' affiliations ....................

Correspondence to: Dr E Isolauri, Department of Paediatrics, Turku University Central Hospital, 20520 Turku, Finland; erika.isolauri@ utu.fi

Revised version received 10 June 2004 Accepted for publication 19 June 2004
B ringing empirical observations ad fontes advances science. In astrophysics, the term "black hole" was introduced to describe an extremely dense star which had collapsed into a singularity under its own gravity. A black hole radiates nothing; it absorbs all matter and energy falling within its sphere. The name was coined only after revisiting the initial theoretical achievements of Karl Schwarzschild, when observations made outside the earth's atmosphere gave astrophysicists empirical $x$ ray data on a new type of cosmic object. In allergology, in contrast, adherence to a paradigm whereby allergy is defined by the presence of specific IgE antibodies has hampered disentanglement. As a result, allergy remains a dubiously defined term with no unambiguous empirical content or explanatory power. The time has come to seize upon the available empirical data and plunge into the original theory of Clemens von Pirquet.

The term allergy was introduced by von Pirquet to denote a changed immunological reactivity which manifests itself on second exposure to an antigen (reviewed by Kay ${ }^{1}$ ). This altered reactivity is uncommitted, giving no indication of the direction of change; equally harmful and protective immune reactivity reflects prior encounter (see fig 1). In modern terms, altered reactivity can be seen to evince either the most common mode of immune response elicited by the intestinal immune system, tolerance, recently defined as any mechanism by which a potentially injurious immune response is prevented, suppressed, or shifted to a non-injurious class of immune response, ${ }^{2}$ or abrogation of such an actively maintained process, which is currently linked to immunoinflammatory disease. Reassessment of the original theory of allergy is important as it would appear that it is not the immunological resources gained during antigen exposure, measurable by specific antibodies or specifically responding lymphocytes, which are decisive for the presence or absence of disease, but the complex cascade of events determining their use.

The notion of food allergy in irritable bowel syndrome (IBS) resurfaces in scientific thinking in this issue of $G u t^{3}$ on the basis of a solid randomised placebo controlled trial conducted by Atkinson and colleagues (see page 1459). Determination of serum IgG antibodies against foods was used to guide the construction of elimination diets.

The presence of specific IgG class antibodies is often accepted as uncommitted or protective "altered reactivity", unlike those of the IgE class. Detection of antigen specific IgE is invariably taken as an attribute of causality, a condition called "IgE mediated disease" and, more specifically, of "allergy". ${ }^{1}$ However, empirical data are accumulating to suggest that transient increases in antigen specific IgE antibodies prevail in most healthy asymptomatic children during the first five years of life. ${ }^{4}$ Secondly, generation of these antibodies (sensitisation) on antigen exposure may not necessarily induce clinical disease (atopic disease). ${ }^{5}$ Thirdly, reducing the risk of atopic disease does not necessitate reduction of sensitisation $^{6-8}$ and, finally, resolution or aggravation of clinical disease is not invariably associated with a corresponding alteration in antibody concentration. Bearing these limitations in mind, however, the clinician may successfully profit from determination of specific IgE to complete the clinical history in an attempt to identify potential offending antigens in a symptomatic patient's diet for the explicit diagnostic elimination-challenge procedure. ${ }^{9}$ This is precisely what Atkinson et al did, with specific IgG antibodies. ${ }^{3}$ They identified a significant reduction in IBS symptom severity in patients on elimination diets, provided that dietary elimination was based on foods against which the individual had raised IgG antibodies; fully compliant patients showed the best clinical improvement. The reverse pattern was observed after reintroduction of the respective foods.

"IBS appears to result from an interplay between susceptibility genes and impaired gut barrier functions, immunological dysregulation, together with bacterial and viral infections and other environmental factors"

In common with allergic disease, IBS appears to result from an interplay between susceptibility

Abbreviations: IBS, irritable bowel syndrome; PRR, pattern recognition receptor 


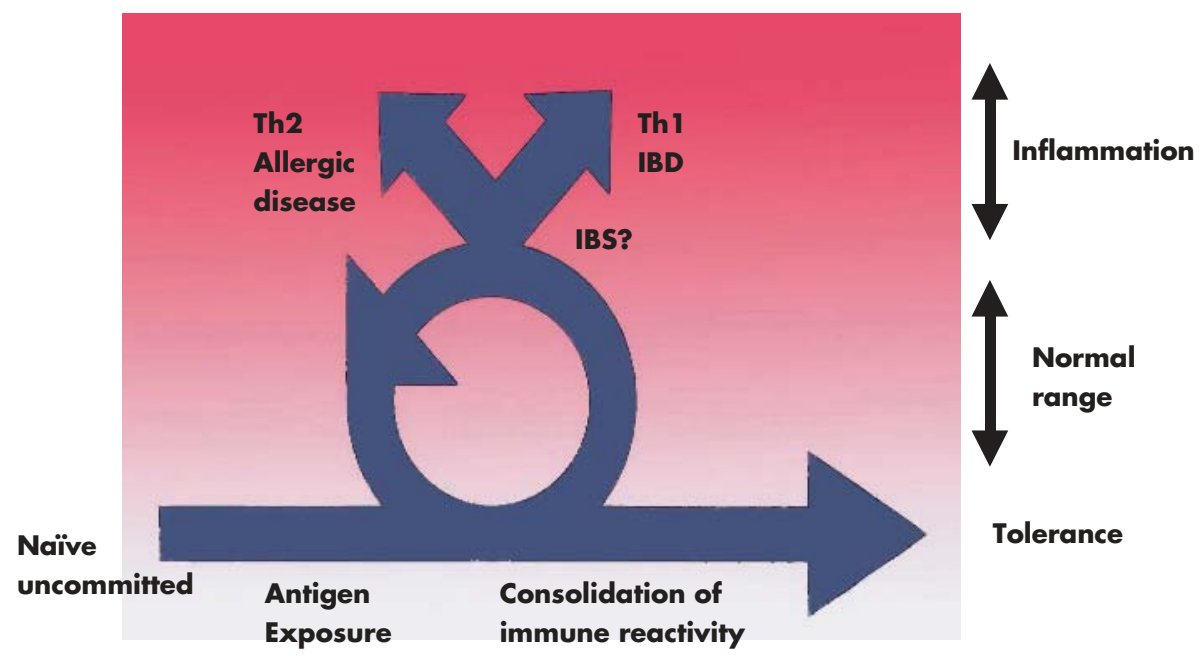

Figure 1 An attempt to reformulate the original conception of the immunological basis of allergy. On exposure to dietary and microbial antigens, the naïve immune system matures either to establish appropriate immune reactivity and tolerance or, in the case of lack of suppressive and regulatory signals, develops inflammatory disease expressed as Th1 skewed autoimmune reactivity (for example, inflammatory bowel disease (IBD)) or Th2 skewed allergic disease. The exact nature of immune reactivity in irritable bowel syndrome (IBS) on this axis remains elusive.

genes and impaired gut barrier functions, immunological dysregulation, together with bacterial and viral infections and other environmental factors. It is no easy matter to describe succinctly "gut barrier function". In the gastrointestinal tract, the external and internal environments are in close proximity. The dilemma of the mucosal surface of the intestine is to fend off the constant challenge from antigens, such as microorganisms, in mounting a brisk response to pathogens, and to enable assimilation of innocuous antigens derived from food. In order to perform these opposing functions, the intestine is in a state of continuous immune responsiveness, and a delicate balance is generated and maintained between concomitant facilitation and suppression of inflammatory responses.

Gut barrier function consists of physiological and immunological factors which exclude and degrade antigens and restrict their adherence, penetration, and transfer. Antigen presenting cells, and more precisely dendritic cells, are pivotal in directing mucosal immune responses. ${ }^{10-12}$ Three dendritic cell derived signals are required for an effective $\mathrm{T}$ cell response. ${ }^{11}$ The nature of signal 1 depends on the antigen in question and its processing; necessary costimulatory molecules create the second signal and the pericellular cytokine milieu is the basis of the third. On antigen recognition, maturation of dendritic cells and secretion of cytokines and chemokines occur. These secretions direct the polarisation of a naïve $\mathrm{T}$ helper cell to type 1 , type 2 , or a regulatory $\mathrm{T}$ cell and thus regulate other adaptive immune responses, such as B cell derived immunoglobulin production. ${ }^{11}$ Tolerance to lumenal dietary and microbial antigens is likely to be achieved through those dendritic cells which induce production of regulatory $\mathrm{T}$ cells secreting interleukin 10 and transforming growth factor $\beta .^{13}$ These cytokines promote gut barrier function by suppressing the production of both T helper 1 and 2 cytokines, ${ }^{14}$ overexpression of which is associated with increased gut permeability. ${ }^{15}{ }^{16}$ Moreover, the anergic $\mathrm{T}$ cells induced by interleukin 10 exposed dendritic cells appear to be able to suppress other T cells in an antigen specific manner. ${ }^{17}$ Transforming growth factor $\beta$ downregulates both $\mathrm{T}$ helper 1 and 2 responses directly ${ }^{18}$ and indirectly by modulating the activity of antigen presenting cells $^{19}$ and favouring the development of regulatory T cells. ${ }^{20}$ After intestinal priming, these cells migrate to the periphery, thus mediating peripheral tolerance on reactivation. In addition to its effects on T cell function, transforming growth factor $\beta$ is a key factor in $\operatorname{IgA}$ production $^{21}$ and thus contributes to maintenance of gut barrier function and to immune responses at other mucosal surfaces also. Taken together, "gut barrier function" strongly depends on antigen processing and presentation and the cytokine milieu in the mucosal immune system, and determines the nature of the immune response (that is, tolerance or inflammation) elicited to a particular antigen.

\section{"Inflammation can cause profound alterations in the function of smooth muscle and enteric nerves as well as in deeper neuromuscular layers"}

In certain circumstances, such as metabolic stress, the peaceful coexistence across the barrier is disturbed and an inflammatory response ensues. ${ }^{22}$ Abrogated barrier function of the gut mucosa leads to greater antigen transfer when the routes of transport are also altered, thereby evoking aberrant immune responses and release of proinflammatory cytokines with further impairment of barrier function. Inflammation can cause profound alterations in the function of smooth muscle and enteric nerves as well as in deeper neuromuscular layers. ${ }^{23}$ Indeed, a subtle inflammatory response and exaggerated sensitivity to that type of response has been suggested to be causative in IBS. In view of recently reported alterations in the immunological defence in IBS, ${ }^{24}$ the trigger(s) of the vicious circle can be depicted among the intraluminal antigens.

In this issue of Gut, Atkinson and colleagues ${ }^{3}$ describe IgG antibody responses to dietary antigens of clinical significance and an apparent causal relation to symptoms in IBS, in a fashion resembling the elimination-challenge procedure in food allergy. To broaden this concept, it is intriguing to speculate that IBS may perhaps also be associated with IgG antibodies against other intraluminal antigens such as those from the indigenous microbiota, partially analogously to loss of tolerance to gut microbiota in inflammatory bowel disease. ${ }^{25} 26$

The human gastrointestinal tract harbours a complex collection of microorganisms which form the individual microbiota typical for each person. Defence is facilitated by peristalsis, secretion of mucus and antimicrobial peptides such as defensins and cathelicidins, and commensal induced 
IgA. ${ }^{27} 28$ Intestinal epithelial cells further contribute to the homeostasis of gut barrier function by a scarcity of both pattern recognition receptors (PRRs; for example, toll-like receptors and nucleotide binding oligomerisation domain proteins) and their coreceptors, expression of active negative regulators of PRR signalling, and secretion of the suppressive cytokines interleukin 10 and transforming growth factor $\beta .^{13}$ All of these characteristics assist in preventing unnecessary and even hazardous systemic immunity to commensals while allowing local protective mucosal immune responses. In addition, some specific strains of non-pathogenic bacteria have been shown to attenuate intestinal inflammation by selective inhibition of intracellular signalling pathways elicited by diverse potentially deleterious stimuli. ${ }^{29}$ A healthy gut microbiota is thus an indispensable component of "gut barrier function".

"A healthy gut microbiota is thus an indispensable component of gut barrier function"

The findings of Atkinson and colleagues ${ }^{3}$ should encourage studies dissecting the mechanisms responsible for IgG production against dietary antigens and their putative role in IBS. This may serve not only IBS research but also that into allergy and allergic diseases. In the perspectives of both normal gut barrier function and the vague findings in a few studies of probiotic supplementation in IBS, ${ }^{31-33}$ we suggest that the possible role of the gut microbiota in the pathogenesis of IBS may deserve closer attention. If the host-microbe cross talk is misinterpreted in IBS, a working target for novel therapeutic interventions beyond elimination diets could be provided in modulating the composition and/or activity of the gut microbiota and promoting gut immune defence. Research interest in the science of nutrition is directed towards improvement of defined physiological functions beyond the nutritional impact of food. The search for active non-nutritive compounds is also a focus for research in the treatment and prevention of allergic diseases.

\section{Authors' affiliations}

E Isolauri, S Rautava, Department of Paediatrics, University of Turku and Turku University Central Hospital, Finland

M Kalliomäki, Massachusetts General Hospital East, Combined Program in Pediatric Gastroenterology and Nutrition, Charlestown,

Massachusetts, USA

\section{REFERENCES}

1 Kay AB. Concepts of allergy and hypersensitivity. In: Kay AB, ed. Allergy and allergic diseases. Oxford: Blackwell Science Ltd, 1997:23-35.

2 Weiner HL. Oral tolerance: immune mechanisms and the generation of Th3 type TGF-beta-secreting regulatory cells. Microbes Infect 2001;3:947-54.

3 Atkinson W, Sheldon TA, Shaath N, et al. Food elimination based on lgG antibodies in irritable bowel symdrome: a randomised controlled trial. Gut 2004; 53: 1459-64.

4 Kulig M, Bergmann R, Klettke U, et al. Natural course of sensitization to food and inhalant allergens during the first 6 years of life. J Allergy Clin Immunol 1999; 103:1173-9.
5 Lau S, Illi S, Sommerfeld C, et al. Early exposure to house-dust mite and cat allergens and development of childhood asthma: a cohort study. Lancet 2000;356:1392-7.

6 Kalliomäki M, Salminen S, Kero $P$, et al. Probiotics in the primary prevention of atopic disease: a randomised, placebo-controlled trial. Lancet 2001;357:1076-9

7 Riedler J, Braun-Fahrländer C, Eder W, et al. Exposure to farming in early life and development of asthma and allergy: a cross-sectional survey. Lancet 2001;358:1129-33

8 Jones CA, Holloway JA, Popplewell EJ, et al. Reduced soluble CD14 levels in amniotic fluid and breast milk are associated with the subsequent development of atopy, eczema, or both. J Allergy Clin Immunol 2002;109:858-66.

9 Bock SA. Diagnostic evaluation. Pediatrics 2003;111:1638-44.

10 Pulendran B, Banchereau J, Maraskovsky E, et al. Modulating the immune response with dendritic cells and their growth factors. Trends Immunol $2001 ; 22: 41-7$.

11 Kapsenberg ML. Dendritic-cell control of pathogen-driven T-cell polarization. Nat Rev Immunol 2003;3:984-93.

12 Stagg AJ, Hart AL, Knight SC, et al. The dendritic cell: its role in intestinal inflammation and relationship with gut bacteria. Gut 2003;52:1522-9.

13 Nagler-Anderson C, Bhan AK, et al. Control freaks: immune regulatory cells. Nat Immunol 2004;5:119-22.

14 Rissoan MC, Soumelis V, Kadowaki N, et al. Reciprocal control of T helper cell and dendritic cell differentiation. Science 1999;283:1183-6.

15 Heyman M, Darmon N, Dupont C, et al. Mononuclear cells from infants allergic to cow's milk secrete tumor necrosis factor $\alpha$, altering intestinal function. Gastroenterology 1994;106:1514-23.

16 Berin MC, Yang PC, Ciok L, et al. Role for IL-4 in macromolecular transport across human intestinal epithelium. Am J Physiol 1999;276:C1046-52.

17 Steinbrink K, Graulich E, Kubsch S, et al. CD4(+) and CD8(+) anergic T cells induced by interleukin-10-treated human dendritic cells display antigenspecific suppressor activity. Blood 2002:99:2468-76.

18 Lúdviksson BR, Seegers D, Resnick AS, et al. The effect of TGF-betal on immune responses of naive versus memory CD4+ Th1/Th2 T cells. Eur J Immunol 2000;30:2101-11.

19 Takeuchi M, Alard P, Streilein JW. TGF-beta promotes immune deviation by altering accessory signals of antigen-presenting cells. J Immunol 1998; 160:1589-97.

20 Toms C, Powrie F. Control of intestinal inflammation by regulatory T cells. Microbes Infect 2001;3:929-35.

21 Stavnezer J. Regulation of antibody production and class switching by TGFbeta. J Immunol 1995; 155: 1647-51.

22 Nazli A, Yang PC, Jury J, et al. Epithelia under metabolic stress perceive commensal bacteria as a threat. Am J Pathol 2004;164:947-57.

23 Collins SM, Piche T, Rampal P. The putative role of inflammation in the irritable bowel syndrome. Gut 2001;49:743-5.

24 Gonsalkorale WM, Perrey C, Pravica V, et al. Interleukin 10 genotypes in irritable bowel syndrome: evidence for an inflammatory component? Gut 2003;52:91-3.

25 Duchmann R, Kaiser I, Hermann E, et al. Tolerance exists towards resident intestinal flora but is broken in active inflammatory bowel disease (IBD). Clin Exp Immunol 1995; 102:448-55

26 Lodes MJ, Cong Y, Elson CO, et al. Bacterial flagellin is a dominant antigen in Crohn disease. J Clin Ivest 2004;113:1296-306.

27 Otte J-M, Kiehne K, Herzig K-H. Antimicrobial peptides in innate immunity of the human intestine. J Gastroenterol 2003;38:717-26.

28 Macpherson AJ, Uhr T. Induction of protective lgA by intestinal dendritic cells carrying commensal bacteria. Science 2004;303:1662-5.

29 Haller D, Jobin C. Interaction between resident luminal bacteria and the host: can a healthy relationship turn sour? J Pediatr Gastroenterol Nutr 2004;38: 123-36.

30 Kelly D, Campbell Jl, King TP, et al. Commensal anaerobic gut bacteria attenuate inflammation by regulating nuclear-cytoplasmic shuttling of PPAR- $\gamma$ and RelA. Nat Immunol 2004;5:104-12.

31 Nobaek S, Johansson M-L, Molin G, et al. Alteration of intestinal microflora is associated with reduction in abnormal bloating and pain in patients with irritable bowel syndrome. Am J Gastroenterol 2000;95:1231-8.

32 Sen S, Mullan MM, Parker TJ, et al. Effect of Lactobacillus plantarum 299v on colonic fermentation and symptoms of irritable bowel syndrome. Dig Dis Sci 2002;47:2615-20.

$33 \mathrm{Kim}$ HJ, Camilleri M, McKinzie S, et al. A randomized controlled trial of a probiotic, VSL\#3, on gut transit and symptoms in diarrhoea-predominan irritable bowel syndrome. Aliment Pharmacol Ther 2003;17:895-904. 